\title{
Alterações retinianas apresentadas em pacientes portadores de hemoglobinopatia falciforme atendidos em um Serviço Universitário de Oftalmologia
}

\author{
Retinal manifestations in patients with sickle cell disease referred to a University Eye Hospital \\ Luiz Guilherme Azevedo de Freitas ${ }^{1}$, David Leonardo Cruvinel Isaac ${ }^{1}$, William Thomas Tannure ${ }^{1}$, Elisa Vieira da Silva Lima², Murilo Batista Abud ${ }^{1}$, \\ Renato Sampaio Tavares ${ }^{2}$, Clovis Arcoverde de Freitas ${ }^{3}$, Marcos Pereira de Ávila ${ }^{4}$
}

\section{RESUMO}

Objetivos: Identificar os principais achados fundoscópicos em pacientes portadores de anemia falciforme encaminhados a um Serviço Oftalmológico de Referência em Goiânia (GO).

Métodos: Foram realizados exames oftalmológicos em 50 pacientes (100 olhos) portadores de hemoglobinopatia falciforme para observar quais as alterações retinianas mais comuns nesse grupo.

Resultados: $\mathrm{O}$ tipo de hemoglobinopatia mais encontrado foi o SS, seguido pelas hemoglobinopatias SC, AS e Sthal. Dentro da amostra estudada, 22\% apresentaram alterações retinianas. Destes $73 \%$ eram do sexo masculino. A alteração retinianas encontradas foram: "sea fan", "black sunburst", hemorragia vítrea e descolamento de retina. Em relação à classificação da retinopatia, $73 \%$ apresentaram a forma proliferativa, sendo vista nos tipos AS e SC e 27\% apresentaram a forma não-proliferativa da retinopatia, sendo vista nos portadores do tipo SS.

Conclusão: Foi observado elevado número de pacientes com alterações retinianas na amostra estudada, sendo o maior número em portadores da hemoglobinopatia SC, seguido dos grupos AS e SS. As alterações proliferativas foram as mais observadas. Hemorragia vítrea e descolamento de retina foram as manifestações proliferativas de maior prevalência e mostraram ser mais frequente em portadores da hemoglobinopatia SC na população estudada.

Descritores: Anemia falciforme; Hemoglobina falciforme; Retina; Descolamento retiniano; Hemorragia vítrea

\begin{abstract}
Purpose: To identify retinal manifestations in patients with sickle cell disease referred to a reference eye hospital in Goiânia (GO).

Methods: Ophthalmic evaluation was made in 50 patients (100 eyes) with sickle cell disease to evaluate the most common manifestations of this group.

Results: Hemoglobinopathy SS was the most commonly found, followed by hemoglobin SC, AS and Stahl. Twenty-two percent of the patients had retinal changes, of these $73 \%$ weremale. Retinal changes observed were: "seafan", "blacksunburst", vitreous hemorrhage, and retinal detachment. In the classification of retinopathy, $73 \%$ had proliferative form, seen in the types AS and SC and 27\% had non-proliferative retinopathy, seen in patients with SS type.

Conclusions: We observed a large numbers of patients with retinal changes, most of them with hemoglobinopathy SC, followed by AS and SS groups. The proliferative changes werethemostcommonlyobserved. Vitreous hemorrhageandretinaldetachment were the most prevalent manifestations in proliferative retinopathy and showed to be more common in patients with SC hemoglobinopathy in the studied population.
\end{abstract}

Keywords: Sickle cell disease; Hemoglobin sickle; Retina; Retinal detachment; Vitreous hemorrhage

\section{INTRODUÇÃO}

As hemoglobinopatias são um grupo de doenças hereditárias descritas pela primeira vez, em 1910, por Herrick, quando investigava pacientes da raça negra portadores com anemia, dor abdominal e artralgia ${ }^{(1,2)}$.

A anemia falciforme é causada por uma mutação no gene da globina beta da hemoglobina, originando uma hemoglobina anormal denominada hemoglobina S (HbS), em vez da hemoglobina A $(\mathrm{HbA})^{(3)}$. Esta mutação causa a substituição de um aminoácido ácido glutâmico por uma valina na posição 6 da cadeia beta, provocando uma alteração físico-química na molécula da hemoglobina. Estas moléculas geneticamente modificadas estão susceptíveis a um processo de polimerização, com a capacidade de alterarem a forma das hemácias (falcização) em situações específicas de mudança de pH, o que ocasiona um encurtamento da vida média dos glóbulos vermelhos. A falcização pode desencadear um processo de vaso-oclusão, culminando em episódios dolorosos e de lesão a órgãos. As alterações retinianas relacionadas à anemia falciforme geralmente ocorrem devido a este processo de oclusão vascular ${ }^{(4,5)}$.

A retinopatia falciforme pode ser dividida em não-proliferativa e proliferativa ${ }^{(6,7)}$. Na retinopatia não-proliferativa os achados mais característicos são: hemorragias pré-retinianas do tipo "salmon patch" e hiperpigmentações retinianas tipo "black sunburst". A forma proliferativa por sua vez, foi classificada segundo achados clínicos em cinco
Submitted for publication: March 22,2011

Accepted for publication:September 15, 2011

Study carried out at Centro de Referência em Oftalmologia do Hospital das Clínicas, Universidade Federal de Goiás - UFG - Goiânia (GO), Brazil.

${ }^{1}$ Physician, Departamento de Retina e Vítreo no Centro de Referência em Oftalmologia do Hospital das Clínicas, Universidade Federal de Goiás - UFG - Goiânia (GO), Brazil.

2 Physician, Setor de Hematologia do Hospital das Clínicas, Universidade Federal de Goiás - UFG Goiânia (GO), Brazil.

3 Physician, Serviço de Uveítes do Hospital São Geraldo, Universidade Federal de Minas Gerais UFMG - Belo Horizonte (MG), Brazil.

${ }^{4}$ Professor, Departamento de Oftalmologia, Universidade Federal de Goiás - UFG - Goiânia (GO), Brazil.
Funding: No specific financial support was available for this study.

Disclosure of potential conflicts of interest: L.G.A.de Freitas, None; D.L.C.Isaac, None; W.T. Tannure, None; E.V.S.Lima, None; M.B.Abud, None; R.S.Tavares, None; C.A.de Freitas, None; M.P.de Ávila, None.

Correspondence address: Luiz Guilherme Azevedo de Freitas. Av. T-2, 401 - Setor Bueno - Goiânia (GO) - 74210-010 - Brazil - E-mail: luizgfreitas@gmail.com 
estágios ${ }^{(8)}$ : 1 - oclusão arteriolar periférica; 2 - anastomoses arteriovenosas periféricas; 3 - proliferações neovasculares e fibrosas; 4 hemorragia vítrea; 5 - descolamento de retina ${ }^{(8)}$

Na retina, a fisiopatologia do processo neovascular iniciar-se-ia devido à oclusão arteriolar periférica com consequente rearranjo dos capilares adjacentes ${ }^{(8)}$. Esses capilares poderiam se dilatar e formar anastomoses arteriovenosas nas áreas entre a retina vascularizada e a isquêmica. A neovascularização surgiria assim a partir da retina vascularizada em direção à avascular, a partir dessas anastomoses. Inicialmente, os neovasos são planos e possuem uma conformação denominada "sea fan", nome dado devido a comparação ao formato em leque de uma espécie de coral marinho (Gorgonia flabellum).

As formações neovasculares podem sangrar levando à hemorragia vítrea (HV) e/ou estão associadas a proliferação fibroglial em direção ao vítreo. As consecutivas hemorragias suscitam a formação de um tecido fibrovascular que pode provocar tração sobre a retina ocasionando eventual descolamento de retina tracional (DRT) ${ }^{(8)}$.

Estudos mostram que alterações fundoscópicas são mais frequentes nos tipos SC e menos frequentes nos tipos SS(5,9). Determinou-se também que a forma proliferativa é quatro vezes mais prevalente na forma SC do que na forma $\mathrm{SS}^{(9)}$.

Pacientes portadores da hemoglobinopatia do tipo SS apresentam doença sistêmica mais grave e com alterações fundoscópicas mais brandas comparadas às alterações encontradas nos portadores do tipo SC. Acredita-se que isso se deve ao fato de os portadores do tipo SS possuirem menor viscosidade sanguínea, devido à anemia mais grave, que funcionaria como efeito protetor contra processos oclusivos na retina. Como pacientes do tipo SC apresentam quadro sistêmico moderado, a viscosidade sanguínea é maior do que os do tipo SS, predispondo a processos oclusivos mais frequentemente $\mathrm{e}^{(9)}$

Esse estudo tem o objetivo de identificar os principais achados fundoscópicos em pacientes portadores de anemia falciforme atendidos em um Serviço Oftalmológico de Referência.

\section{MÉTODOS}

O presente estudo foi aprovado pelo Comitê de Ética em Pesquisa Médica Humana e Animal do Hospital da Clínicas (CEPMHA-HC), da Universidade Federal de Goiás (UFG). Foi realizado estudo transversal no Centro de Referência em Oftalmologia (CEROF) do HC da UFG no período de junho de 2010 a dezembro de 2010, onde foram examinados 48 pacientes encaminhados do Departamento de Hematologia do HC da UFG para exame de rotina (com diagnóstico de hemoglobinopatia falciforme previamente confirmado) e 2 pacientes que foram encaminhados da urgência oftalmológica do mesmo serviço com sinais e sintomas oculares sugestivos de retinopatia falciforme. $\mathrm{O}$ tipo de hemoglobinopatia foi confirmado por eletroforese de hemoglobina em $\mathrm{pH}$ alcalino. Os pacientes foram submetidos à anamenese e exame oftalmológico completo, com oftalmoscopia binocular indireta sobre midríase. Angiografia fluoresceínica e ecografia ocular foram realizadas conforme a indicação de cada caso.

Foi utilizado um formulário como protocolo de pesquisa que continha: identificação, idade, sexo, raça, tipo de hemoglobinopatia, melhor acuidade visual corrigida, pela tabela de Snellen a 5 metros (pacientes acima de 5 anos de idade), tonometria de aplanação com o tonômetro de Goldmann, biomicroscopia do segmento anterior em lâmpada de fenda, fundo do olho com oftalmoscópio indireto, angiografia fluoresceínica (nos casos em que houvesse suspeita de alteração retiniana) e ecografia ocular (em opacidades de meios).

Todos os pacientes ou responsáveis legais leram e assinaram termo de consentimento livre e esclarecido mostrando-se cientes de estarem participando do estudo. Os critérios de inclusão foram: pacientes que fossem encaminhados do Departamento de Hematologia do HC/UFG deveriam apresentar hemoglobinopatia falci- forme diagnosticada pelo teste de eletroforese de hemoglobina; apresentar idade entre 2 e 60 anos e assinar termo de consentimento livre e esclarecido.

Os critérios de exclusão foram: idade fora dos padrões préestabelecidos; opacidades corneana ou catarata que impedissem a visibilidade adequada da retina; doenças retinianas que não a retinopatia falciforme; e recusa, por parte do paciente, de participar do estudo.

Foram incluídos dois pacientes que apresentaram suspeita de retinopatia falciforme atendidos na urgência oftalmológica e que, após solicitação do teste de eletroforese de hemoglobina, os diagnósticos de hemoglobinopatia foram confirmados. Nenhum paciente foi excluído do estudo. Todos os pacientes portadores de alterações retinianas indicativas de tratamento foram submetidos à suas respectivas condutas.

\section{RESULTADOS}

Foram estudados 50 pacientes (100 olhos). Dos pacientes estudados 28 eram do sexo masculino (56\%). O tipo de hemoglobinopatia mais encontrada foi a do tipo SS com 31 pacientes (62\%), seguida pela hemoglobinopatia SC com 14 pacientes (28\%), AS com 4 pacientes (10\%) e Sthal com 1 paciente (2\%). Dentro da amostra estudada, 11 pacientes (22\%) apresentaram alterações retinianas. Destes 8 eram do sexo masculino (73\%) e 3 do sexo feminino (27\%) (Tabela 1)

Alterações retinianas foram diagnosticadas em 3 pacientes com o tipo SS, em 5 pacientes portadores do tipo SC e em 3 dos pacientes portadores do tipo AS. As alterações retinianas encontradas foram: hemorragias pré-retinianas do tipo "salmon patch" em 1 paciente (1 olho); "black sunburst" em 5 pacientes (7 olhos); "sea fan" presente em 3 pacientes (4 olhos); hemorragia vítrea em 4 pacientes (4 olhos) e descolamento de retina em 4 pacientes (4 olhos). Dois pacientes (2 olhos) do grupo SC apresentaram história pregressa de vitrectomia via pars plana para HV e DR. Os achados divididos nos respectivos grupos encontram-se listados na tabela 2.

Em relação à classificação da retinopatia, 3 pacientes $(36,4 \%)$ apresentaram a forma não-proliferativa da retinopatia, sendo 3 pacientes portadores do tipo SS e 1 do tipo AS e 7 pacientes $(63,6 \%)$ apresentaram a forma proliferativa, sendo 2 pacientes com o tipo AS e $5 \mathrm{com}$ o tipo SC. O paciente portador do tipo SThal não apresentou nenhum tipo de alteração na retina.

\section{DISCUSSÃO}

O presente estudo avaliou pacientes com hemoglobinopatias consecutivamente atendidos em um hospital escola. Na amostra

Tabela 1. Incidência das hemoglobinopatias

\begin{tabular}{lcc}
\hline Tipo & Número pacientes & Porcentagem \\
\hline SS & 31 & 62 \\
SC & 14 & 28 \\
AS & 4 & 8 \\
SThal & 1 & 2 \\
\hline
\end{tabular}

Tabela 2. Achados fundoscópicos de acordo com as hemoglobinopatias

\begin{tabular}{lccccc}
\hline Alteração retiniana & SC & SS & AS & SThal & Total \\
\hline "Salmon patch" & 1 & 0 & 0 & 0 & 1 \\
"Black sunburst" & 1 & 3 & 1 & 0 & 5 \\
"Sea fun" & 2 & 0 & 1 & 0 & 3 \\
Hemorragia vítrea & 2 & 0 & 2 & 0 & 4 \\
Descolamento de retina & 3 & 1 & 0 & 0 & 4 \\
\hline
\end{tabular}


estudada evidenciou-se maior prevalência da hemoglobinopatia do tipo SS, representando 62\% da amostra, seguida da SC com 28\%, AS com $8 \%$ e Sthal com 2\% corroborando com resultados de outros estudos previamente publicados ${ }^{(2,10,11)}$. As alterações do segmento posterior foram classificadas em proliferativas e não-proliferativas, onde foram encontrados 4 pacientes na forma não-proliferativa, sendo todos eles 3 do grupo SS e 1 do AS e 7 pacientes na forma proliferativa, sendo 5 no grupo SC e 2 no grupo AS.

Dentre os achados classificados como não-proliferativos foram encontrados: hemorragias pré-retinianas do tipo "salmon patch" em 1 paciente (1 olho) no grupo SC; pontos hiperpigmentados subretinianos ("black sunburst"), observados em 5 pacientes, 3 no grupo SS, 1 no AS e 1 no SC.

As manifestações da retinopatia falciforme proliferativa (RFP) encontradas foram: "sea fan", hemorragia vítrea e descolamento de retina. Os pacientes portadores da hemoglobinopatia SC mostraram prevalência de retinopatia falciforme proliferativa. Tais achados foram semelhantes aos de outros trabalhos já publicados na literatura, demonstrando ser este o principal grupo afetado. Estudo realizado em Acra, capital da República do Gana, na África ocidental, demonstrou maior incidência da doença proliferativa da retina em portadores do genótipo SC. Foram examinados 201 pacientes com hemoglobinopatia falciforme, sendo 87 portadores do tipo SC. Destes 25,3\% apresentavam retinopatia falciforme proliferativa, resultados esses semelhantes aos encontrados no presente estudo (31,7\% de portadores do tipo SC com RFP) $)^{(4,5,10,12)}$.

Foi verificado um número elevado de pacientes apresentando hemorragia vítrea e descolamento de retina, estudos já relataram a presença dessas manifestações em portadores de hemoglobinopatia SC, porém não com tamanha frequência ${ }^{(13,14)}$.

Dois pacientes, um do grupo SC e um do grupo AS, que apresentaram retinopatia falciforme proliferativa, somente receberam o diagnóstico da hemoglobinopatia após investigação hematológica solicitada pelo oftalmologista, a partir de suspeita clínica da doença baseado nos achados encontrados ao exame fundoscópico. Com isso, mostramos que através do exame oftalmológico de rotina pode-se, em casos específicos, se estabelecer suspeita clínica da doença sistêmica a partir de alterações oculares específicas.

Dois pacientes do grupo SC apresentaram história pregressa de cirurgia vitreorretiniana. Um deles operado devido a DR (no exame do estudo foi observado DR recorrente em funil fechado) e o outro pela presença de HV (Figura 1).

Os pacientes com HV e DR (2 do grupo AS, 1 do grupo SC) foram submetidos a tratamento cirúrgico (Figura 1). Um paciente do grupo SC que apresentava HV não retornou até o final desse estudo para tratamento específico e outros três casos de DR não apresentavam mais indicação de cirurgia.

\section{CONCLUSÃO}

Foi observado elevado número de pacientes com alterações retinianas na amostra estudada (22\%) sendo o maior número em portadores da hemoglobinopatia SC, seguido dos grupos AS e SS. As alterações proliferativas foram as mais frequentes $(63,6 \%$ dos

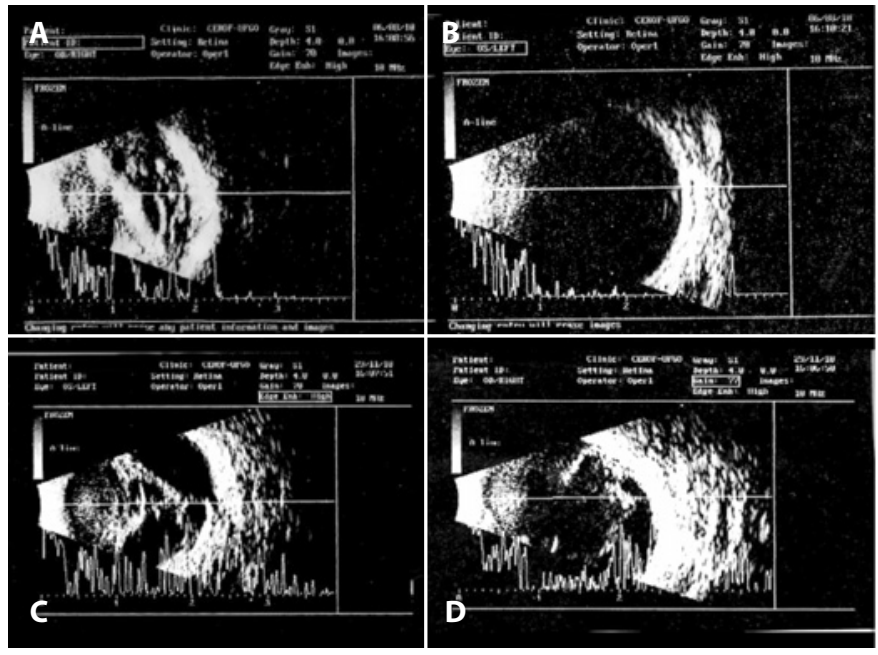

Figura 1. A e C) Descolamento de retina antigo; B) Olho submetido a cirurgia de hemorragia vítrea; D) Hemorragia vítrea.

que apresentaram alterações retinianas) estando presente em todos do grupo SC e em 2 do grupo AS. Hemorragia vítrea e descolamento de retina obtiveram a maior prevalência nas alterações proliferativas, mostrando ser mais frequente em portadores da hemoglobinopatia SC na população estudada.

\section{REFERÊNCIAS}

1. Herrick JB. Peculiar elongated and sickle-shaped red blood corpuscles in a case of severe anemia. 1910.Yale J Biol Med. 2001;74(3):179-84.

2. Oliveira FVP, Aihara T, Cançado RD. Alterações fundoscópicas nas hemoglobinopatias SS e SC. Arq Bras Oftalmol. 1996;59(3):234-8.

3. Manual de diagnóstico e tratamento de doenças falciformes. Brasília: ANVISA; 2001.

4. Clarkson JG. The ocular manifestations of sickle-cell disease: a prevalence and natural history study. Trans Am Ophthalmol Soc. 1992;90:481-504

5. Bonanomi MT, Cunha SL, de Araújo JT. Fundoscopic alterations in SS and SC hemoglobinopathies. Study of Brazilian population. Ophthalmologica. 1988;197(1):26-33.

6. Gonçalves JCM, Braga JAP, Nione AS, Simoceli RA, Yamamoto M. Retinopatia falciforme em crianças. Arq Bras Oftalmol. 1990;53(4):158-61.

7. Bonanomi MTBC, Oliveira AA, Suzuki H. Hemoglobinopatias. In: Abujamra S, Ávila M, Barsante C I. Retina e vítreo. São Paulo: Roca; 2000; p.592-601.

8. Goldberg MF. Classification and pathogenesis of proliferative sickle retinopathy. Am J Ophthalmol. 1971;71(3):649-65.

9. Bisol T, Fior O, Esteves JF, Friderich JR. Influência do genótipo da hemoglobinopatia falciforme nas manifestações retinianas em pacientes de um hospital universitário. Arq Bras Oftalmol. 2000;63(4):273-6.

10. Osafo-Kwaako A, Kimani K, llako D, Akafo S, Ekem I, Rodrigues O, et al. Ocular manifestations of sickle cell disease at the Korle-bu Hospital, Accra, Ghana. Eur J Ophthalmol. 2010. [Epub ahead of print].

11. Akinsola FB, Kehinde MO. Ocular findings in sickle cell disease patients in Lagos. Niger Postgrad Med J. 2004;11(3):203-6.

12. Cury D, Boa-Sorte N, Lyra IM, Zanette AD, Castro-Lima H, Galvão-Castro B, Gonçalves MS. Ocular lesions in sickle cell disease patients from Bahia, Brazil. Rev Bras Oftalmol. 2010; 69(4):259-63 